\title{
Stability of Slopes for Dam Excavation by Slope Mass Rating in the Pare Hydroelectric Project
}

\author{
Sylvia Kashyap \\ Department of Civil Engineering \\ Assam Engineering College \\ Guwahati, India \\ Ranjeet Bahadur Singh \\ Department of Civil Engineering \\ Assam Engineering College \\ Guwahati, India \\ Pragati Goswami \\ Department of Civil Engineering \\ Assam Engineering College \\ Guwahati, India
}

\author{
Diganta Goswami \\ Department of Civil Engineering \\ Assam Engineering College \\ Guwahati, India \\ Pawan Kumar Singh \\ Department of Civil Engineering \\ Assam Engineering College \\ Guwahati, India \\ Dibyajyoti Kalita \\ Department of Civil Engineering \\ Assam Engineering College \\ Guwahati, India
}

\begin{abstract}
Dam is an important element in all river valley projects, and excavation of both banks of the river, where the dam is abutted, is a very important consideration for the total cost of the project. Thus, the stability analysis of the side slopes of the rock mass is a very important exercise, for a feasible hydro-electric power project. The study area lies in the Papunpare district of Arunachal Pradesh, India which is represented by Upper Siwalik sub-group of the SubHimalayan range. In this paper the stability of rock slopes for a stable excavation for dam construction, is analyzed with the help of Slope Mass Rating (SMR) by using Basic Rock Mass Rating. Support measures, where ever required has also been recommended based on SMR. The satisfactory performance of the excavated slopes at the dam site of Pare H.E. P. project establishes that SMR method can be used with confidence for rock slope stability analysis.
\end{abstract}

Keywords-SMR; RMRbasic; adjustment factors; stability

\section{INTRODUCTION}

Concrete gravity dam of a Hydro Electric Power project is abutted to the fresh rocks on both banks of the river. A stable, yet the most economical slope, needs to be designed for the purpose. In this paper, Slope Mass Rating system (Romana, 1985) as in [1] is used to study the stability of the rock slopes for abutting the dam of the Pare H.E.P. project at Papunpare district of Arunachal Pradesh. The study area lies within the upper Siwalik sub-group of the SubHimalayan range and are characterized by soft tertiary sedimentary sandstone/ siltstone. Four drill holes, namely, DAH 2, 3,4 \& 5, drilled by NEEPCO Ltd. along the dam axis, exhibits repetitive sequence of coarse to fine grained, greyish coloured, soft, friable, moderately fresh sandstone, pebble impregnated sandstone, sand rock and pebble beds, on either banks. Geology of the dam site, as mapped by geologists of NEEPCO Ltd. are presented in the following sub-section.

\section{GEOLOGY OF DAM SITE}

The left and right rocky abutment was indentified with three sets of joint oriented in different directions. The dip/strike of the joint sets are given in the table I.

TABLE I. DIP/STRIKE OF JOINT SETS

\begin{tabular}{|c|c|c|c|c|}
\hline \multirow{2}{*}{ Joints } & \multicolumn{2}{|c|}{ Left Abutment } & \multicolumn{2}{c|}{ Right Abutment } \\
\cline { 2 - 5 } & Dip & Strike & Dip & Strike \\
\hline $\mathrm{J} 1$ & $60^{\circ}-75^{\circ}$ & $\mathrm{N} 235^{\circ}-250^{\circ}$ & $52^{\circ}-70^{\circ}$ & $\mathrm{N} 230^{\circ}-240^{\circ}$ \\
\hline $\mathrm{J} 2$ & $45^{\circ}-75^{\circ}$ & $\mathrm{N} 150^{\circ}-180^{\circ}$ & $28^{\circ}-46^{\circ}$ & $\mathrm{N} 140^{\circ}-170^{\circ}$ \\
\hline $\mathrm{J} 3$ & $32^{\circ}$ & $\mathrm{N} 195^{\circ}$ & $32^{\circ}$ & $\mathrm{N} 195^{\circ}$ \\
\hline
\end{tabular}

\section{ROCK MASS CLASSIFICATION}

In a river valley project, excavation of a dam requires the modified rock slopes to be stable. The improper excavation methods on natural slope conditions and modification of cut slopes can develop new cracks which may lead to instability of the rock slope. Therefore the preliminary approach of a dam excavation should include the understanding of the geological and geomechanical parameters to prevent slope failure during and after excavation.

For various engineering designs and stability analysis, Rock Mass Classification systems form the cornerstone of empirical design approach and are extensively implemented in rock engineering. Rock Mass Classifications have been recently employed in underground constructions, tunneling and in mining projects (Barton 1974, 1988; Bieniawski 1989; Laubscher 1990) as in [2][3][4]. A rock mass is characterized by comparing the values obtained from visual inspection or by simple tests with some standard sets of descriptions and values for some specific parameters. Thus with the help of standard qualitative data and guidelines, rock masses described by different geologists and engineers at different locations can be compared and analyzed. 


\section{A. Rock Mass Rating System}

This classification system (developed by Bieniawski in 1973, [5]) can be applied to a given rock mass that is divided into a number of geological structural regions where each region represents separate geological structural unit. Generally the boundary of the units coincides with a change in the rock type or with structural feature such as fault. The following are the six representing parameters (given by Bieniawski in 1989) which are determined for each unit:

1) Uniaxial Compressive Strength (UCS) of intact rock material

2) Rock Quality Designation (RQD)

3) Joint or Discontinuity spacing

4) Joint condition

5) Groundwater condition

6) Joint orientation

All the above mentioned parameters can be obtained from field and can also be measured from borehole data. The ratings of these six parameters are summarized and the RMR value is obtained. As the dam block is divided into nine blocks, the basic RMR is derived for each of the blocks.

a) Uniaxial Compressive Strength of Intact Rock Material:Table II shows the rating of the parameter Uniaxial Compressive Strength which is calculated using the relation of principal stresses.

TABLE II. UNIAXIAL COMPRESSIVE STRENGTH OF INTACT ROCK MATERIAL

\begin{tabular}{|c|c|c|c|c|c|}
\hline $\begin{array}{c}\text { Friction } \\
\text { angle } \\
(\varphi)\end{array}$ & $\begin{array}{c}\mathbf{C} \text { in } \\
\mathbf{K N} / \mathbf{m}^{2}\end{array}$ & $\begin{array}{c}\text { UCS in } \\
\mathbf{K N} / \mathbf{m}^{2}\end{array}$ & \multicolumn{2}{|c|}{ UCS in Mpa } & Rating \\
\hline $48^{\circ}$ & 16.1 & 838.84 & 0.838 & $\begin{array}{c}\text { Extremely } \\
\text { Weak }\end{array}$ & 0 \\
\hline
\end{tabular}

b) Rock Quality Designation:Table III presents the rating corresponding to the average RQD value.

TABLE III. ROCK QUALITY DESIGNATION (RQD) FROM BOREHOLES

\begin{tabular}{|c|c|c|c|}
\hline Borehole & RQD (\%) & Average RQD (\%) & Rating \\
\hline DAH2 & 20 & & \\
DAH3 & 16 & \multirow{2}{*}{18.75} & 3 \\
\hline DAH4 & 21 & & \\
\hline DAH5 & 18 & & \\
\hline
\end{tabular}

c) Discontinuity Spacing: There are many set of discontinuities present in different dam blocks, some ranging from very closely spaced to moderately spaced while some are randomly spaced. Ratings are given accordingly for each discontinuity set occurring on the rock mass (Table IV).

d) Discontinuity Condition: This parameter includes continuity, aperture, weathering of the planes of weakness or the wall rock, roughness of discontinuity surfaces and infilling materials present in the discontinuities. From the geology obtained from dam sites, the ratings for each of the conditions have been calculated for each dam blocks and are found to fall under the range of 19-25. This implies that the rock mass is of mixed quality conditions as both good and poor quality prevails.

e) Groundwater Condition: The general hydraulic condition derived from this site varies from moist to dripping.

Table IV shows the computation of the corresponding values of basic RMR of the three joint sets from the ratings of the parameters. The basic RMR values are computed for dam block 1 and 9 as it falls in left and right abutment of the dam site respectively.

TABLE IV. BASIC RMR OBTAINED FROM THE RATINGS OF THE PARAMETERS

\begin{tabular}{|c|c|c|c|c|c|c|c|}
\hline $\begin{array}{c}\text { Dam } \\
\text { Block } \\
\text { Nos. }\end{array}$ & $\begin{array}{c}\text { Join } \\
\mathbf{t} \\
\text { sets/ } \\
\text { Bed } \\
\text { ding }\end{array}$ & $\begin{array}{l}\mathbf{R} \\
\mathbf{Q} \\
\mathbf{D}\end{array}$ & $\begin{array}{l}\mathrm{U} \\
\mathrm{C} \\
\mathrm{S}\end{array}$ & $\begin{array}{c}\text { Disconti } \\
\text { nuity } \\
\text { spacing }\end{array}$ & $\begin{array}{l}\text { Discontinu } \\
\text { ity } \\
\text { condition }\end{array}$ & $\begin{array}{c}\text { Ground } \\
\text { water } \\
\text { conditio } \\
\text { n }\end{array}$ & $\begin{array}{c}\mathbf{R} \\
\mathbf{M} \\
\mathbf{R} \\
\text { ba } \\
\text { sic }\end{array}$ \\
\hline Dam & J1 & 3 & 0 & 5 & 21 & 7 & 36 \\
\hline Block & $\mathrm{J} 2$ & 3 & 0 & 5 & 22 & 7 & 37 \\
\hline No. 1 & $\mathrm{~J} 3$ & 3 & 0 & 5 & 25 & 7 & 40 \\
\hline Dam & $\mathrm{J} 1$ & 3 & 0 & 10 & 19 & 7 & 39 \\
\hline Block & $\mathrm{J} 2$ & 3 & 0 & 15 & 23 & 7 & 48 \\
\hline No. 9 & $\mathrm{~J} 3$ & 3 & 0 & 5 & 23 & 7 & 38 \\
\hline
\end{tabular}

The above $\mathrm{RMR}_{\text {basic }}$ values obtained can be used to calculate the Slope Mass Rating values which will help to predict the stablility of the rock slope.

\section{B. Slope Mass Rating (SMR) System}

Slope Mass Rating system has become the most extensively used classification system for rock slopes. The SMR system is the extension of the basic Rock Mass Rating along with four adjustment factors that take into account the geometrical relationship between the rock slope face and discontinuity affecting rock mass (factors $F_{1}$ to $F_{3}$ ) as well as the excavation method used $\left(\mathrm{F}_{4}\right)$.Romana (1985) derived SMR system for the purpose of assessment of rock slope stability, from the studies of natural and cut slopes along the roads. It is obtained using the following expression:

$\mathbf{S M R}=\mathbf{R M R}_{\mathrm{b}}+\left(\mathbf{F}_{1} \cdot \mathbf{F}_{2} \cdot \mathbf{F}_{3}\right)+\mathbf{F}_{4}$

Where $\mathrm{RMR}_{\mathrm{b}}$ is the basic RMR obtained from Bieniawski's Rock Mass Classification system without any correction.

$F_{1}$ depends upon the parallelism between discontinuity, $\alpha_{j}$ (and the plunge direction of line of intersection, $\alpha_{i}$ in case of wedge failure) and slope dip direction. It ranges from 0.15 to 1.0. Initially the value of $F_{1}$ was established and was found approximately equal to $(1-\sin \mathrm{A})^{2}$, where A represents the angle between slope strike and joint strike.

$F_{2}$ depends on the discontinuity $\operatorname{dip}(\beta \mathrm{j})$ in the case of planar failure and $\beta \mathrm{i}$ (or plunge) of the intersection line in wedge failure. Its value also varies from 0.15 to 1.0. For toppling failure, $F_{2}$ is 1.0. Empirically, $F_{2}=\tan \beta_{\mathrm{j}}$

$F_{3}$ depends on the relationship between slope dip $(\beta s)$ and discontinuity dip ( $\beta \mathrm{j}$ ) (for toppling or planar failure cases) or the plunge of line of intersection ( $\beta i$ ) (wedge failure case). 
$\mathrm{F}_{4}$ is a correction factor that depends on the excavation method used.

For the evaluation of the adjustment factors, some dip angles of the slope face are considered and with the help of dip/strike of the discontinuities all the factors are calculated for the rock masses. The strike of the dam axis on left bank is $\mathrm{N} 74^{\circ} \mathrm{E}$ so the slope strike for the left abutment is found to be $\mathrm{N} 164^{\circ}$ and strike of the dam axis on right bank is $\mathrm{S} 74^{\circ} \mathrm{W}$ and thus the slope strike for right abutment is found to be $\mathrm{N} 344^{\circ}$. According to the formula proposed by Romana (1985), all the adjustment factors have been calculated.

1) Adjustment Factor $F_{l}$ : Using the formula $\mathbf{F}_{\mathbf{1}}=\mid \boldsymbol{\alpha}_{\mathrm{j}}$ $\boldsymbol{\alpha}_{\mathrm{s}}$, where $\alpha_{\mathrm{j}}$ is the joint strike and $\alpha_{\mathrm{s}}$ is the slope strike, it has been observed that for slope strike $\mathrm{N} 164^{\circ}$ on the left bank and $\mathrm{N} 344^{\circ}$ on the right bank of Pare river orientation is very favorable with respect to the strikes of the joint sets in most of the considered cases except for joint strike ranging from $\mathrm{N} 150^{\circ}$ to $\mathrm{N} 180^{\circ}$ present on the left rocky abutment with slope strike $\mathrm{N} 164^{\circ}$ which refers to fair condition. This represents that overall both the slopes are stable with respect to adjustment factor $\mathrm{F}_{1}$.

2) Adjustment Factor $F_{2}$ : As $F_{2}$ depends on the joint dip angle, it has been observed that only for joint dip $28^{\circ}$ and $32^{\circ}$ the factor $F_{2}$ comes out to be favorable and fair respectively. For other joint dips greater than $45^{\circ}, \mathrm{F}_{2}$ represents very unfavorable condition. This refers that the slope may undergo planar failure.

3) Adjustment Factor $F_{3}$ : As the adjustment factor $F_{3}$ refers to the relationship between joint dip and slope dip, the values of $\mathrm{F}_{3}$ are obtained for various slope dip with respect to the three joint sets present on both the abutments.

For the left abutment a number of slope dip $(\beta s)$ are considered ranging from $45^{\circ}$ upto $75^{\circ}$ and $F_{3}$ is calculated for each joint sets as shown in Table $\mathrm{V}$.
Similarly in the right abutment certain slope dips are considered ranging for $45^{\circ}-75^{\circ}$, and $\mathrm{F}_{3}$ is calculated for each of the joint sets as shown in Table VI.

4) Ajustment Factor $F_{4}$ : This adjustment factor is related to the method of excavation which includes natural slope, or the cut slope excavated by pre-splitting, smooth blasting, normal blasting, poor blasting and mechanical excavation.

As per the information gathered from the agency it has been known that mainly pre-splitting is used while excavating the slopes. Value of the adjustment factor $F_{4}$ for pre-splitting is +10 as proposed by Romana (1985).

\section{5) Calculation of Slope Mass Rating:}

The Slope Mass Ratings are calculated for the rocky abutments situated on the either banks of the river Pare where joint sets and bedding planes are present using the formula as in (1).

For the ease of the calculation, only the extreme values of the range of dip and strike of a joint set has been considered for which SMR values are obtained and rest of the angles under that range are considered to fall under that particular range of SMR.

a) SMR for left abutment: Here, all the SMR values for the rock mass present on the left bank of river Pare have been calculated and the failure modes have been determined for each of the joint sets with respect to the considered slope dip angles as shown in Table V.

b) SMR for right abutment: Similarly, all the SMR values for the rock mass present on the right bank of river Pare have been calculated and the failure modes have been determined for each of the joint sets as in Table VI.

TABLE V: CALCULATION OF SMR VALUE USING DIFFERENT SLOPE DIPS FOR LEFT ABUTMENT

\begin{tabular}{|c|c|c|c|c|c|c|c|c|c|c|c|c|}
\hline \multicolumn{13}{|c|}{ CALCULATION OF SMR VALUE USING SLOPE DIP $45^{\circ}$} \\
\hline \multirow{2}{*}{$\begin{array}{l}\text { Joint } \\
\text { sets }\end{array}$} & \multirow{2}{*}{$\begin{array}{l}\text { Slope } \\
\text { dip } \\
\left(\boldsymbol{\beta}_{s}\right)\end{array}$} & \multirow{2}{*}{$\begin{array}{c}\text { Slope } \\
\text { Strike } \\
\left(\alpha_{s}\right)\end{array}$} & \multirow{2}{*}{$\begin{array}{c}\text { Joint dip } \\
\left(\boldsymbol{\beta}_{j}\right)\end{array}$} & \multirow{2}{*}{$\begin{array}{c}\text { Joint } \\
\text { strike }\left(\alpha_{j}\right)\end{array}$} & \multirow[b]{2}{*}{$F_{1}$} & \multirow[b]{2}{*}{$F_{2}$} & \multicolumn{2}{|c|}{$F_{3}$} & \multirow[b]{2}{*}{$F_{4}$} & \multirow[b]{2}{*}{$R M R_{\text {basic }}$} & \multirow[b]{2}{*}{$S M R$} & \multirow[b]{2}{*}{ Failure mode } \\
\hline & & & & & & & $\beta_{j}-\beta_{s}$ & Rating & & & & \\
\hline \multirow[b]{2}{*}{$\mathrm{J} 1$} & \multirow{5}{*}{$45^{\circ}$} & \multirow[b]{2}{*}{$\mathrm{N} 164^{\circ}$} & $\operatorname{Min} 60^{\circ}$ & \multirow[b]{2}{*}{$\begin{array}{l}\mathrm{N} 235^{\circ}- \\
250^{\circ}\end{array}$} & 0.15 & 1 & $60-45=15^{\circ}$ & 0 & 10 & \multirow[b]{2}{*}{36} & 46 & \multirow{2}{*}{$\begin{array}{l}\text { Planar along some } \\
\text { joints and many } \\
\text { wedges }\end{array}$} \\
\hline & & & $\operatorname{Max} 75^{\circ}$ & & 0.15 & 1 & $75-45=30^{\circ}$ & 0 & 10 & & 46 & \\
\hline \multirow[t]{2}{*}{$\mathrm{J} 2$} & & \multirow[t]{2}{*}{$\mathrm{N} 164^{\circ}$} & $\operatorname{Min} 45^{\circ}$ & \multirow{2}{*}{$\begin{array}{l}\mathrm{N} 150^{\circ}- \\
180^{\circ}\end{array}$} & 0.7 & 0.85 & $45-45=0^{\circ}$ & -25 & 10 & \multirow[t]{2}{*}{37} & 32.125 & $\begin{array}{c}\text { Planar or big } \\
\text { wedges }\end{array}$ \\
\hline & & & $\operatorname{Max} 75^{\circ}$ & & 0.7 & 1 & $75-45=30^{\circ}$ & 0 & 10 & & 47 & \multirow{2}{*}{$\begin{array}{l}\text { Planar along some } \\
\text { joints and many } \\
\text { wedges }\end{array}$} \\
\hline $\mathrm{J} 3$ & & $\mathrm{~N} 164^{\circ}$ & $32^{\circ}$ & $\mathrm{N} 195^{\circ}$ & 0.15 & 0.7 & $32-45=-13^{\circ}$ & -60 & 10 & 40 & 43.7 & \\
\hline \multicolumn{13}{|c|}{ CALCULATION OF SMR VALUE USING SLOPE DIP 50 ${ }^{\circ}$} \\
\hline \multirow[b]{2}{*}{$\mathrm{J} 1$} & \multirow{5}{*}{$50^{\circ}$} & \multirow[b]{2}{*}{$\mathrm{N} 164^{\circ}$} & Min $60^{\circ}$ & \multirow[b]{2}{*}{$\begin{array}{l}\mathrm{N} 235^{\circ}- \\
250^{\circ}\end{array}$} & 0.15 & 1 & $60-50=10^{\circ}$ & -6 & 10 & \multirow[b]{2}{*}{36} & 45.1 & \multirow{2}{*}{$\begin{array}{c}\text { Planar along some } \\
\text { joints and many } \\
\text { wedges }\end{array}$} \\
\hline & & & $\operatorname{Max} 75^{\circ}$ & & 0.15 & 1 & $75-50=25^{\circ}$ & 0 & 10 & & 46 & \\
\hline \multirow[t]{2}{*}{$\mathrm{J} 2$} & & \multirow[t]{2}{*}{$\mathrm{N} 164^{\circ}$} & $\operatorname{Min} 45^{\circ}$ & \multirow{2}{*}{$\begin{array}{l}\mathrm{N} 150^{\circ}- \\
180^{\circ}\end{array}$} & 0.7 & 0.85 & $45-50=-5^{\circ}$ & -50 & 10 & \multirow[t]{2}{*}{37} & 17.25 & $\begin{array}{l}\text { Big planar or soil- } \\
\text { like or circular }\end{array}$ \\
\hline & & & $\operatorname{Max} 75^{\circ}$ & & 0.7 & 1 & $75-50=25^{\circ}$ & 0 & 10 & & 47 & \multirow{2}{*}{$\begin{array}{l}\text { Planar along some } \\
\text { joints and many } \\
\text { wedges }\end{array}$} \\
\hline $\mathrm{J} 3$ & & $\mathrm{~N} 164^{\circ}$ & $32^{\circ}$ & $\mathrm{N} 195^{\circ}$ & 0.15 & 0.7 & $32-50=-18^{\circ}$ & -60 & 10 & 40 & 43.7 & \\
\hline
\end{tabular}


TABLE V: Contd.

\begin{tabular}{|c|c|c|c|c|c|c|c|c|c|c|c|c|}
\hline \multicolumn{13}{|c|}{ CALCULATION OF SMR VALUE USING SLOPE DIP 55 } \\
\hline \multirow{2}{*}{$\begin{array}{l}\text { Joint } \\
\text { sets }\end{array}$} & \multirow{2}{*}{$\begin{array}{c}\text { Slope } \\
\text { dip } \\
\left(\boldsymbol{\beta}_{s}\right)\end{array}$} & \multirow{2}{*}{$\begin{array}{c}\text { Slope } \\
\text { Strike } \\
\left(\alpha_{s}\right)\end{array}$} & \multirow{2}{*}{$\underset{\left(\boldsymbol{\beta}_{j}\right)}{\text { Joint dip }}$} & \multirow{2}{*}{$\begin{array}{c}\text { Joint } \\
\text { strike }\left(\alpha_{j}\right)\end{array}$} & \multirow[b]{2}{*}{$F_{1}$} & \multirow[b]{2}{*}{$\boldsymbol{F}_{2}$} & \multicolumn{2}{|l|}{$F_{3}$} & \multirow[b]{2}{*}{$\boldsymbol{F}_{4}$} & \multirow[b]{2}{*}{$\boldsymbol{R M R}_{\text {basic }}$} & \multirow[b]{2}{*}{ SMR } & \multirow[b]{2}{*}{ Failure mode } \\
\hline & & & & & & & $\beta_{j}-\beta_{s}$ & Rating & & & & \\
\hline \multirow[b]{2}{*}{$\mathrm{J} 1$} & \multirow{5}{*}{$55^{\circ}$} & \multirow{2}{*}{$\mathrm{N} 164^{\circ}$} & Min $60^{\circ}$ & \multirow[b]{2}{*}{$\begin{array}{c}\mathrm{N} 235^{\circ}- \\
250^{\circ}\end{array}$} & 0.15 & 1 & $60-55=5^{\circ}$ & -6 & 10 & \multirow[b]{2}{*}{36} & 45.1 & \multirow{2}{*}{$\begin{array}{c}\text { Planar along some } \\
\text { joints and many } \\
\text { wedges }\end{array}$} \\
\hline & & & $\operatorname{Max} 75^{\circ}$ & & 0.15 & 1 & $75-55=15^{\circ}$ & 0 & 10 & & 46 & \\
\hline \multirow[t]{2}{*}{$\mathrm{J} 2$} & & \multirow[t]{2}{*}{$\mathrm{N} 164^{\circ}$} & $\operatorname{Min} 45^{\circ}$ & \multirow{2}{*}{$\begin{array}{c}\mathrm{N} 150^{\circ}- \\
180^{\circ}\end{array}$} & 0.7 & 0.85 & $45-55=-10^{\circ}$ & -50 & 10 & \multirow[t]{2}{*}{37} & 17.25 & $\begin{array}{l}\text { Big planar or soil- } \\
\text { like or circular }\end{array}$ \\
\hline & & & $\operatorname{Max} 75^{\circ}$ & & 0.7 & 1 & $75-55=20^{\circ}$ & 0 & 10 & & 47 & \multirow{2}{*}{$\begin{array}{c}\text { like or circular } \\
\text { Planar along some } \\
\text { joints and many } \\
\text { wedges }\end{array}$} \\
\hline $\mathrm{J} 3$ & & $\mathrm{~N} 164^{\circ}$ & $32^{\circ}$ & $\mathrm{N} 195^{\circ}$ & 0.15 & 0.7 & $32-55=-23^{\circ}$ & -60 & 10 & 40 & 43.7 & \\
\hline \multicolumn{13}{|c|}{ CALCULATION OF SMR VALUE USING SLOPE DIP $60^{\circ}$} \\
\hline \multirow[b]{2}{*}{$\mathrm{J} 1$} & \multirow{5}{*}{$60^{\circ}$} & & $\operatorname{Min} 60^{\circ}$ & $\mathrm{N} 235^{\circ}-$ & 0.15 & 1 & $60-60=0^{\circ}$ & -25 & 10 & & 42.25 & Planar along some \\
\hline & & $\mathrm{N} 164^{\circ}$ & $\operatorname{Max} 75^{\circ}$ & $250^{\circ}$ & 0.15 & 1 & $75-60=15^{\circ}$ & 0 & 10 & 36 & 46 & $\begin{array}{l}\text { joints and many } \\
\text { wedges }\end{array}$ \\
\hline $\mathrm{J} 2$ & & $\mathrm{~N} 164^{\circ}$ & $\operatorname{Min} 45^{\circ}$ & $\mathrm{N} 150^{\circ}-$ & 0.7 & 0.85 & $45-60=-15$ & -60 & 10 & 37 & 11.3 & $\begin{array}{l}\text { Big planar or soil- } \\
\text { like or circular }\end{array}$ \\
\hline & & & $\operatorname{Max} 75^{\circ}$ & & 0.7 & 1 & $75-60=15^{\circ}$ & 0 & 10 & & 47 & Planar along some \\
\hline $\mathrm{J} 3$ & & $\mathrm{~N} 164^{\circ}$ & $32^{\circ}$ & $\mathrm{N} 195^{\circ}$ & 0.15 & 0.7 & $32-60=-28^{\circ}$ & -60 & 10 & 40 & 43.7 & $\begin{array}{c}\text { joints and many } \\
\text { wedges }\end{array}$ \\
\hline & & & & CALC & LATI & NOF S & R VALUE US & G SLOI & DIP & & & \\
\hline & & & $\operatorname{Min} 60^{\circ}$ & & 0.15 & 1 & $60-65=-5^{\circ}$ & -50 & 10 & & 38.5 & $\begin{array}{c}\text { Planar or big } \\
\text { wedges }\end{array}$ \\
\hline $\mathrm{J} 1$ & & $\mathrm{~N} 164^{\circ}$ & $\operatorname{Max} 75^{\circ}$ & $\begin{array}{l}N 235^{\circ}- \\
250^{\circ}\end{array}$ & 0.15 & 1 & $75-65=10^{\circ}$ & -6 & 10 & 36 & 45.1 & $\begin{array}{c}\text { Planar along some } \\
\text { joints and many } \\
\text { wedges }\end{array}$ \\
\hline $\mathrm{J} 2$ & $65^{\circ}$ & $\mathrm{N} 164^{\circ}$ & $\operatorname{Min} 45^{\circ}$ & $\begin{array}{l}\mathrm{N} 150^{\circ}- \\
180^{\circ}\end{array}$ & 0.7 & 0.85 & $45-65=-20^{\circ}$ & -60 & 10 & 37 & 11.3 & $\begin{array}{l}\text { Big planar or soil- } \\
\text { like or circular }\end{array}$ \\
\hline & & & $\operatorname{Max} 75^{\circ}$ & & 0.7 & 1 & $75-65=10^{\circ}$ & -6 & 10 & & 42.8 & Planar along some \\
\hline $\mathrm{J} 3$ & & $\mathrm{~N} 164^{\circ}$ & $32^{\circ}$ & $\mathrm{N} 195^{\circ}$ & 0.15 & 0.7 & $32-65=-33$ & -60 & 10 & 40 & 43.7 & $\begin{array}{c}\text { joints and many } \\
\text { wedges }\end{array}$ \\
\hline & & & & CALC & JLAT & NOFS & $\overline{\text { R VALUE US }}$ & G SLOI & DIP & & & \\
\hline & & & $\operatorname{Min} 60^{\circ}$ & & 0.15 & 1 & $60-70=-10^{\circ}$ & -50 & 10 & & 38.5 & $\begin{array}{c}\text { Planar or big } \\
\text { wedges }\end{array}$ \\
\hline $\mathrm{J} 1$ & $70^{\circ}$ & $\mathrm{N} 164^{\circ}$ & $\operatorname{Max} 75^{\circ}$ & $\begin{array}{l}\mathrm{N} 235^{\circ}- \\
250^{\circ}\end{array}$ & 0.15 & 1 & $75-70=5^{\circ}$ & -6 & 10 & 36 & 45.1 & $\begin{array}{c}\text { Planar along some } \\
\text { joints and many } \\
\text { wedges }\end{array}$ \\
\hline $\mathrm{J} 2$ & 10 & $\mathrm{~N} 164^{\circ}$ & $\operatorname{Min} 45^{\circ}$ & $\mathrm{N} 150^{\circ}-$ & 0.7 & 0.85 & $45-70=-25^{\circ}$ & -60 & 10 & 37 & 11.3 & $\begin{array}{l}\text { Big planar or soil- } \\
\text { like or circular }\end{array}$ \\
\hline & & & $\operatorname{Max} 75^{\circ}$ & & 0.7 & 1 & $75-70=5^{\circ}$ & -6 & 10 & & 42.8 & Planar along some \\
\hline $\mathrm{J} 3$ & & $\mathrm{~N} 164^{\circ}$ & $32^{\circ}$ & $\mathrm{N} 195^{\circ}$ & 0.15 & 0.7 & $32-70=-38$ & -60 & 10 & 40 & 43.7 & $\begin{array}{c}\text { joints and many } \\
\text { wedges }\end{array}$ \\
\hline & & & & CALC & JLAT & N OFS & R VALUE US & G SLOI & DIP & & & \\
\hline & & & $\operatorname{Min} 60^{\circ}$ & & 0.15 & 1 & $60-75=-15^{\circ}$ & -60 & 10 & & 37 & $\begin{array}{c}\text { Planar or big } \\
\text { wedges }\end{array}$ \\
\hline $\mathrm{J} 1$ & & $\mathrm{~N} 164^{\circ}$ & $\operatorname{Max} 75^{\circ}$ & $\begin{array}{l}\mathrm{N} 235^{\circ}- \\
250^{\circ}\end{array}$ & 0.15 & 1 & $75-75=0^{\circ}$ & -25 & 10 & 36 & 42.25 & $\begin{array}{c}\text { Planar along some } \\
\text { joints and many } \\
\text { wedges }\end{array}$ \\
\hline J2 & $75^{\circ}$ & $\mathrm{N} 164^{\circ}$ & $\operatorname{Min} 45^{\circ}$ & $\mathrm{N} 150^{\circ}{ }_{-}$ & 0.7 & 0.85 & $45-75=-30^{\circ}$ & -60 & 10 & 37 & 11.3 & $\begin{array}{l}\text { Big planar or soil- } \\
\text { like or circular }\end{array}$ \\
\hline $\mathrm{J} 2$ & & N164 & $\operatorname{Max} 75^{\circ}$ & $180^{\circ}$ & 0.7 & 1 & $75-75=0^{\circ}$ & -25 & 10 & 31 & 29.5 & $\begin{array}{c}\text { Planar or big } \\
\text { wedges }\end{array}$ \\
\hline $\mathrm{J} 3$ & & $\mathrm{~N} 164^{\circ}$ & $32^{\circ}$ & $\mathrm{N} 195^{\circ}$ & 0.15 & 0.7 & $32-75=-43$ & -60 & 10 & 40 & 43.7 & $\begin{array}{c}\text { Planar along some } \\
\text { joints and many } \\
\text { wedges }\end{array}$ \\
\hline
\end{tabular}

Thus, from the above table it has been observed that the rock mass is partially stable with respect to plane failure except for joint dip $45^{\circ}$ with joint strike ranging from $\mathrm{N} 150^{\circ}$ to $\mathrm{N} 180^{\circ}$ which indicates that the left rocky abutment is completely unstable due to the presence of this joint. Factor $\mathrm{F}_{3}$ comes out to be very unfavorable for joint dip $45^{\circ}$ and also for $32^{\circ}$ having joint strike $\mathrm{N} 195^{\circ}$ for all the considered slope dip angles. While, as the slope dip increases, the rating of $\mathrm{F}_{3}$ decreases and thus in turn increases the instability of the rock slope. When we consider toppling mode of failure the rock mass comes out to be favorable. In Table VII the stability criteria of the left rocky abutment along with the failure probability is given with respect to the dip/strike of the discontinuity sets present in the rock mass. 
TABLE VI: CALCULATION OF SMR VALUE USING DIFFERENT SLOPE DIPS FOR RIGHT ABUTMENT

\begin{tabular}{|c|c|c|c|c|c|c|c|c|c|c|c|c|}
\hline \multicolumn{13}{|c|}{ CALCULATION OF SMR VALUE USING SLOPE DIP $45^{\circ}$} \\
\hline \multirow{2}{*}{$\begin{array}{l}\text { Joint } \\
\text { sets }\end{array}$} & \multirow{2}{*}{$\begin{array}{c}\text { Slope } \\
\text { dips } \\
\left(\boldsymbol{\beta}_{s}\right) \\
\end{array}$} & \multirow{2}{*}{$\begin{array}{c}\text { Slope } \\
\text { Strike } \\
\left(\alpha_{s}\right)\end{array}$} & \multirow{2}{*}{$\underset{\left(\beta_{j}\right)}{\text { Joint dip }}$} & \multirow{2}{*}{$\begin{array}{c}\text { Joint strike } \\
\left(\alpha_{j}\right)\end{array}$} & \multirow[b]{2}{*}{$F_{1}$} & \multirow[b]{2}{*}{$F_{2}$} & \multicolumn{2}{|c|}{$F_{3}$} & \multirow[b]{2}{*}{$\boldsymbol{F}_{4}$} & \multirow[b]{2}{*}{$\boldsymbol{R M R _ { \text { basic } }}$} & \multirow[b]{2}{*}{ SMR } & \multirow{2}{*}{$\begin{array}{c}\text { Failure } \\
\text { mode }\end{array}$} \\
\hline & & & & & & & $\beta_{j}-\beta_{s}$ & Rating & & & & \\
\hline \multirow{2}{*}{$\mathrm{J} 1$} & \multirow{5}{*}{$45^{\circ}$} & \multirow{2}{*}{$\mathrm{N} 344^{\circ}$} & $\operatorname{Min} 52^{\circ}$ & $\mathrm{N} 230^{\circ}-$ & 0.15 & 1 & $52-45=7^{\circ}$ & -6 & 10 & \multirow{2}{*}{39} & 48.1 & \multirow{5}{*}{$\begin{array}{c}\text { Planar } \\
\text { along } \\
\text { some } \\
\text { joints } \\
\text { and } \\
\text { many } \\
\text { wedges } \\
\end{array}$} \\
\hline & & & $\operatorname{Max} 70^{\circ}$ & $240^{\circ}$ & 0.15 & 1 & $70-45=25^{\circ}$ & 0 & 10 & & 49 & \\
\hline \multirow{2}{*}{$\mathrm{J} 2$} & & \multirow{2}{*}{$\mathrm{N} 344^{\circ}$} & $\operatorname{Min} 28^{\circ}$ & $\mathrm{N} 140^{\circ}-$ & 0.15 & 0.4 & $28-45=-17^{\circ}$ & -60 & 10 & \multirow{2}{*}{48} & 54.4 & \\
\hline & & & $\operatorname{Max} 46^{\circ}$ & $170^{\circ}$ & 0.15 & 1 & $46-45=1^{\circ}$ & -6 & 10 & & 57.1 & \\
\hline $\mathrm{J} 3$ & & $\mathrm{~N} 344^{\circ}$ & $32^{\circ}$ & $\mathrm{N} 195^{\circ}$ & 0.15 & 0.7 & $32-45=-13^{\circ}$ & -60 & 10 & 38 & 41.7 & \\
\hline \multicolumn{13}{|c|}{ CALCULATION OF SMR VALUE USING SLOPE DIP 50 ${ }^{\circ}$} \\
\hline \multirow{2}{*}{$\mathrm{J} 1$} & \multirow{5}{*}{$50^{\circ}$} & $\mathrm{N} 344^{\circ}$ & $\operatorname{Min} 52^{\circ}$ & $\mathrm{N} 230^{\circ}-$ & 0.15 & 1 & $52-50=2^{\circ}$ & -6 & 10 & 30 & 48.1 & Planar \\
\hline & & N 344 & $\operatorname{Max} 70^{\circ}$ & $240^{\circ}$ & 0.15 & 1 & $70-50=20^{\circ}$ & 0 & 10 & 39 & 49 & along \\
\hline & & & $\operatorname{Min} 28^{\circ}$ & $\mathrm{N} 140^{\circ}-$ & 0.15 & 0.4 & $28-50=-22^{\circ}$ & -60 & 10 & & 54.4 & some \\
\hline $\mathrm{J} 2$ & & $\mathrm{~N} 344^{\circ}$ & $\operatorname{Max} 46^{\circ}$ & $170^{\circ}$ & 0.15 & 1 & $46-50=-4^{\circ}$ & -50 & 10 & 48 & 50.5 & joints \\
\hline $\mathrm{J} 3$ & & $\mathrm{~N} 344^{\circ}$ & $32^{\circ}$ & $\mathrm{N} 195^{\circ}$ & 0.15 & 0.7 & $32-50=-18^{\circ}$ & -60 & 10 & 38 & 41.7 & $\begin{array}{c}\text { many } \\
\text { wedges }\end{array}$ \\
\hline & & & & ALCULAT & N OF & IR V & JE USING SL & E DIP 5 & & & & \\
\hline I & & $N 344^{\circ}$ & $\operatorname{Min} 52^{\circ}$ & $\mathrm{N} 230^{\circ}-$ & 0.15 & 1 & $52-55=-3^{\circ}$ & -50 & 10 & 30 & 41.5 & Planar \\
\hline$J_{1}$ & & 10544 & $\operatorname{Max} 70^{\circ}$ & $240^{\circ}$ & 0.15 & 1 & $70-55=15^{\circ}$ & 0 & 10 & 39 & 49 & along \\
\hline & & & Min $28^{\circ}$ & $\mathrm{N} 140^{\circ}{ }_{-}$ & 0.15 & 0.4 & $28-55=-27^{\circ}$ & -60 & 10 & & 54.4 & some \\
\hline$\sqrt{2}$ & $55^{\circ}$ & $\mathrm{N} 344^{\circ}$ & $\operatorname{Max} 46^{\circ}$ & $170^{\circ}$ & 0.15 & 1 & $46-55=-9^{\circ}$ & -50 & 10 & 48 & 50.5 & joints \\
\hline $\mathrm{J} 3$ & & $\mathrm{~N} 344^{\circ}$ & $32^{\circ}$ & $\mathrm{N} 195^{\circ}$ & 0.15 & 0.7 & $32-55=-23^{\circ}$ & -60 & 10 & 38 & 41.7 & $\begin{array}{c}\text { many } \\
\text { wedges }\end{array}$ \\
\hline & & & & ALCULAT & N OF & IR V & JE USING SL & E DIP 6 & & & & \\
\hline & & $\mathrm{N} 344^{\circ}$ & $\operatorname{Min} 52^{\circ}$ & $\mathrm{N} 230^{\circ}-$ & 0.15 & 1 & $52-60=-8^{\circ}$ & -50 & 10 & 39 & 41.5 & Planar \\
\hline $\mathrm{J} 1$ & & N344 & $\operatorname{Max} 70^{\circ}$ & $240^{\circ}$ & 0.15 & 1 & $70-60=10^{\circ}$ & -6 & 10 & 39 & 48.1 & along \\
\hline I2 & & $\mathrm{N} 344^{\circ}$ & $\operatorname{Min} 28^{\circ}$ & $\mathrm{N} 140^{\circ}{ }_{-}$ & 0.15 & 0.4 & $28-60=-32^{\circ}$ & -60 & 10 & 48 & 54.4 & some \\
\hline $\mathrm{J} 2$ & $60^{\circ}$ & $\mathrm{N} 344^{\circ}$ & $\operatorname{Max} 46^{\circ}$ & $170^{\circ}$ & 0.15 & 1 & $46-60=-14^{\circ}$ & -60 & 10 & 48 & 49 & joints \\
\hline $\mathrm{J} 3$ & & $\mathrm{~N} 344^{\circ}$ & $32^{\circ}$ & $\mathrm{N} 195^{\circ}$ & 0.15 & 0.7 & $32-60=-28^{\circ}$ & -60 & 10 & 38 & 41.7 & $\begin{array}{c}\text { and } \\
\text { many } \\
\text { wedges }\end{array}$ \\
\hline & & & & ALCULAT & N OF & IR V & JE USING SL & E DIP 6 & & & & \\
\hline $\mathrm{J} 1$ & & $\mathrm{~N} 344^{\circ}$ & $\operatorname{Min} 52^{\circ}$ & $\begin{array}{c}\mathrm{N} 230^{\circ}- \\
240^{\circ}\end{array}$ & 0.15 & 1 & $52-65=-13^{\circ}$ & -60 & 10 & 39 & 40 & $\begin{array}{l}\text { Planar or } \\
\text { big } \\
\text { wedges }\end{array}$ \\
\hline & & & $\operatorname{Max} 70^{\circ}$ & & 0.15 & 1 & $70-65=5^{\circ}$ & -6 & 10 & & 48.1 & Planar \\
\hline I2 & $65^{\circ}$ & $\mathrm{N} 344^{\circ}$ & $\operatorname{Min} 28^{\circ}$ & $\mathrm{N} 140^{\circ}-$ & 0.15 & 0.4 & $28-65=-37^{\circ}$ & -60 & 10 & 48 & 54.4 & along \\
\hline $\mathrm{J}_{2}$ & & N344 & $\operatorname{Max} 46^{\circ}$ & $170^{\circ}$ & 0.15 & 1 & $46-65=-19^{\circ}$ & -60 & 10 & 48 & 49 & some \\
\hline $\mathrm{J} 3$ & & $\mathrm{~N} 344^{\circ}$ & $32^{\circ}$ & $\mathrm{N} 195^{\circ}$ & 0.15 & 0.7 & $32-65=-33^{\circ}$ & -60 & 10 & 38 & 41.7 & $\begin{array}{l}\text { and } \\
\text { many } \\
\text { wedges }\end{array}$ \\
\hline & & & & EALCULAT & N OF & IR V & JE USING SL & E DIP 7 & & & & \\
\hline $\mathrm{J} 1$ & & $\mathrm{~N} 344^{\circ}$ & $\operatorname{Min} 52^{\circ}$ & $\begin{array}{c}\mathrm{N} 230^{\circ}- \\
240^{\circ}\end{array}$ & 0.15 & 1 & $52-75=-23^{\circ}$ & -60 & 10 & 39 & 40 & $\begin{array}{c}\text { Planar or } \\
\text { big } \\
\text { wedges }\end{array}$ \\
\hline & & & $\operatorname{Max} 70^{\circ}$ & & 0.15 & 1 & $70-75=-5^{\circ}$ & -50 & 10 & & 41.5 & Planar \\
\hline & $75^{\circ}$ & $\mathrm{N} 344^{\circ}$ & $\operatorname{Min} 28^{\circ}$ & $\mathrm{N} 140^{\circ}-$ & 0.15 & 0.4 & $28-75=-47^{\circ}$ & -60 & 10 & & 54.4 & along \\
\hline $\mathrm{J} 2$ & 15 & N344 & $\operatorname{Max} 46^{\circ}$ & $170^{\circ}$ & 0.15 & 1 & $46-75=-29^{\circ}$ & -60 & 10 & 48 & 49 & some \\
\hline $\mathrm{J} 3$ & & $\mathrm{~N} 344^{\circ}$ & $32^{\circ}$ & $\mathrm{N} 195^{\circ}$ & 0.15 & 0.7 & $32-75=-43^{\circ}$ & -60 & 10 & 38 & 41.7 & $\begin{array}{l}\text { joints } \\
\text { and } \\
\text { many } \\
\text { wedges }\end{array}$ \\
\hline
\end{tabular}

SMR for the right rocky abutment shows that the rock mass is comparatively better than the left rock mass because of the orientation of the discontinuities for which the ratings of adjustment factors $F_{1}$ and $F_{2}$ came out to be good while for factor $\mathrm{F}_{3}$, ratings are decreased as the slope dip has been increased. As a whole, the right abutment mostly lies in the partially stable category and for each of the three joint sets present in the rock mass in relation to the considered slope dip of $55^{\circ}$ and has been observed to be fair. In Table VIII the stability criteria of the right rocky abutment along with the failure probability is given with respect to the dip/strike of the discontinuity sets present in the rock mass. 
TABLE VII. STABILITY OF THE LEFT ABUTMENT

\begin{tabular}{|c|c|c|c|c|c|c|c|c|c|c|c|}
\hline \multirow[b]{3}{*}{$\begin{array}{c}\text { Slope } \\
\text { dip } \\
\text { angle } \\
\mathbf{s}\left(\boldsymbol{\beta}_{\mathrm{s}}\right)\end{array}$} & \multirow{2}{*}{\multicolumn{4}{|c|}{$\frac{\text { Joint set } 1}{S M R}$}} & \multirow{2}{*}{\multicolumn{4}{|c|}{$\frac{\text { Joint set } 2}{S M R}$}} & \multirow{2}{*}{\multicolumn{2}{|c|}{$\begin{array}{c}\text { Joint set } 3 \\
S M R\end{array}$}} & \multirow{3}{*}{$\begin{array}{c}\text { Failur } \\
e \\
\text { Proba } \\
\text { b-ility } \\
\text { of the } \\
\text { rock } \\
\text { mass } \\
(\%)\end{array}$} \\
\hline & & & & & & & & & & & \\
\hline & $\begin{array}{c}F o r \\
\beta_{j}=60^{\circ} \\
\text { and } \\
a_{j}=N 235 \\
\circ-250^{\circ}\end{array}$ & Stability & $\begin{array}{c}\text { For } \\
\beta_{j}=75^{\circ} \\
\text { and } \\
\alpha_{j}=N 235 \\
{ }^{\circ}-250^{\circ}\end{array}$ & Stability & $\begin{array}{c}\text { For } \beta_{j}=45^{\circ} \\
\text { and } \\
\alpha_{j}=N 150^{\circ}- \\
180^{\circ}\end{array}$ & Stability & $\begin{array}{c}\text { For } \\
\beta_{j}=75^{\circ} \\
\text { and } \\
\alpha_{j}=N 150 \\
\circ-180^{\circ}\end{array}$ & Stability & $\begin{array}{c}\text { For } \beta_{j}=32^{\circ} \\
\text { and } \\
\alpha_{j}=N 195^{\circ}\end{array}$ & Stability & \\
\hline $45^{\circ}$ & 46 & $\begin{array}{c}\text { Partially } \\
\text { stable }\end{array}$ & 46 & $\begin{array}{c}\text { Partially } \\
\text { stable }\end{array}$ & 32.125 & Unstable & 47 & $\begin{array}{c}\text { Partially } \\
\text { stable }\end{array}$ & 43.7 & $\begin{array}{c}\text { Partially } \\
\text { stable }\end{array}$ & 40 \\
\hline $50^{\circ}$ & 45.1 & $\begin{array}{c}\text { Partially } \\
\text { stable }\end{array}$ & 46 & $\begin{array}{c}\text { Partially } \\
\text { stable }\end{array}$ & 17.25 & $\begin{array}{l}\text { Completel } \\
\text { y unstable }\end{array}$ & 47 & $\begin{array}{c}\text { Partially } \\
\text { stable }\end{array}$ & 43.7 & $\begin{array}{c}\text { Partially } \\
\text { stable }\end{array}$ & 40 \\
\hline $55^{\circ}$ & 45.1 & $\begin{array}{c}\text { Partially } \\
\text { stable }\end{array}$ & 46 & $\begin{array}{c}\text { Partially } \\
\text { stable }\end{array}$ & 17.25 & $\begin{array}{l}\text { Completel } \\
\text { y unstable }\end{array}$ & 47 & $\begin{array}{c}\text { Partially } \\
\text { stable }\end{array}$ & 43.7 & $\begin{array}{c}\text { Partially } \\
\text { stable }\end{array}$ & 40 \\
\hline $60^{\circ}$ & 42.25 & $\begin{array}{c}\text { Partially } \\
\text { stable }\end{array}$ & 46 & $\begin{array}{c}\text { Partially } \\
\text { stable }\end{array}$ & 11.3 & $\begin{array}{l}\text { Completel } \\
\text { y unstable }\end{array}$ & 47 & $\begin{array}{c}\text { Partially } \\
\text { stable }\end{array}$ & 43.7 & $\begin{array}{c}\text { Partially } \\
\text { stable }\end{array}$ & 60 \\
\hline $65^{\circ}$ & 38.5 & Unstable & 45.1 & $\begin{array}{c}\text { Partially } \\
\text { stable }\end{array}$ & 11.3 & $\begin{array}{l}\text { Completel } \\
\text { y unstable }\end{array}$ & 42.8 & $\begin{array}{c}\text { Partially } \\
\text { stable }\end{array}$ & 43.7 & $\begin{array}{c}\text { Partially } \\
\text { stable }\end{array}$ & 60 \\
\hline $70^{\circ}$ & 38.5 & Unstable & 45.1 & $\begin{array}{c}\text { Partially } \\
\text { stable }\end{array}$ & 11.3 & $\begin{array}{l}\text { Completel } \\
\text { y unstable }\end{array}$ & 42.8 & $\begin{array}{c}\text { Partially } \\
\text { stable }\end{array}$ & 43.7 & $\begin{array}{c}\text { Partially } \\
\text { stable }\end{array}$ & 60 \\
\hline $75^{\circ}$ & 37 & Unstable & 42.25 & $\begin{array}{c}\text { Partially } \\
\text { stable }\end{array}$ & 11.3 & $\begin{array}{l}\text { Completel } \\
\text { y unstable }\end{array}$ & 29.5 & Unstable & 43.7 & $\begin{array}{c}\text { Partially } \\
\text { stable }\end{array}$ & 90 \\
\hline
\end{tabular}

TABLE VIII. STABILITY OF THE RIGHT ABUTMENT

\begin{tabular}{|c|c|c|c|c|c|c|c|c|c|c|c|}
\hline \multirow[b]{2}{*}{$\begin{array}{l}\text { Slope } \\
\text { dip } \\
\text { angles } \\
\left(\beta_{\mathrm{s}}\right)\end{array}$} & \multicolumn{4}{|c|}{$\begin{array}{c}\text { Joint set 1 } \\
S M R\end{array}$} & \multicolumn{4}{|c|}{$\begin{array}{c}\text { Joint set } 2 \\
S M R\end{array}$} & \multicolumn{2}{|c|}{$\begin{array}{c}\text { Joint set } 3 \\
\text { SMR }\end{array}$} & \multirow{2}{*}{$\begin{array}{c}\text { Failur } \\
e \\
\text { Proba } \\
\text { b-ility } \\
\text { of the } \\
\text { rock } \\
\text { mass } \\
(\%)\end{array}$} \\
\hline & $\begin{array}{c}\text { For } \\
\beta_{j}=52^{\circ} \\
\text { and } \\
\alpha_{j}=230^{\circ}- \\
240^{\circ}\end{array}$ & Stability & $\begin{array}{c}\text { For } \\
\beta_{j}=70^{\circ} \\
\text { and } \\
\alpha_{j}=230^{\circ} \\
-240^{\circ}\end{array}$ & Stability & $\begin{array}{c}\text { For } \beta_{j}=28^{\circ} \\
\text { and } \alpha_{j}=140^{\circ} \\
-170^{\circ}\end{array}$ & Stability & $\begin{array}{c}\text { For } \\
\beta_{j}=46^{\circ} \\
\text { and } \\
\alpha_{j}=140^{\circ} \\
-170^{\circ}\end{array}$ & Stability & $\begin{array}{c}\text { For } \beta_{j}=32^{\circ} \\
\text { and } \\
\alpha_{j}=N 195^{\circ}\end{array}$ & Stability & \\
\hline $45^{\circ}$ & 48.1 & $\begin{array}{c}\text { Partially } \\
\text { stable }\end{array}$ & 49 & $\begin{array}{c}\text { Partially } \\
\text { stable }\end{array}$ & 54.4 & $\begin{array}{c}\text { Partially } \\
\text { stable }\end{array}$ & 57.1 & $\begin{array}{c}\text { Partially } \\
\text { stable }\end{array}$ & 41.7 & $\begin{array}{c}\text { Partially } \\
\text { stable }\end{array}$ & 40 \\
\hline $50^{\circ}$ & 48.1 & $\begin{array}{c}\text { Partially } \\
\text { stable }\end{array}$ & 49 & $\begin{array}{c}\text { Partially } \\
\text { stable }\end{array}$ & 54.4 & $\begin{array}{c}\text { Partially } \\
\text { stable }\end{array}$ & 50.5 & $\begin{array}{c}\text { Partially } \\
\text { stable }\end{array}$ & 41.7 & $\begin{array}{c}\text { Partially } \\
\text { stable }\end{array}$ & 40 \\
\hline $55^{\circ}$ & 41.5 & $\begin{array}{l}\text { Partially } \\
\text { stable }\end{array}$ & 49 & $\begin{array}{l}\text { Partially } \\
\text { stable }\end{array}$ & 54.4 & $\begin{array}{l}\text { Partially } \\
\text { stable }\end{array}$ & 50.5 & $\begin{array}{c}\text { Partially } \\
\text { stable }\end{array}$ & 41.7 & $\begin{array}{l}\text { Partially } \\
\text { stable }\end{array}$ & 40 \\
\hline $60^{\circ}$ & 41.5 & $\begin{array}{l}\text { Partially } \\
\text { stable }\end{array}$ & 48.1 & $\begin{array}{c}\text { Partially } \\
\text { stable }\end{array}$ & 54.4 & $\begin{array}{c}\text { Partially } \\
\text { stable }\end{array}$ & 49 & $\begin{array}{c}\text { Partially } \\
\text { stable }\end{array}$ & 41.7 & $\begin{array}{c}\text { Partially } \\
\text { stable }\end{array}$ & 40 \\
\hline $65^{\circ}$ & 40 & Unstable & 48.1 & $\begin{array}{c}\text { Partially } \\
\text { stable }\end{array}$ & 54.4 & $\begin{array}{c}\text { Partially } \\
\text { stable }\end{array}$ & 49 & $\begin{array}{c}\text { Partially } \\
\text { stable }\end{array}$ & 41.7 & $\begin{array}{c}\text { Partially } \\
\text { stable }\end{array}$ & 40 \\
\hline
\end{tabular}

\section{OBSERVATION}

The rocky abutment present in the left bank of river Pare consists of three set of joints which are oriented in both favorable and unfavorable orientations. Joint set 2 is found to be the most critical joint sets having dip angle ranging from $45^{\circ}$ to $75^{\circ}$. Joints with dip of $45^{\circ}$ produce completely unstable slopes, which require re-excavation (even for slopes with slope dip $45^{\circ}$ ). However, joints with dip $75^{\circ}$ have SMR value above 40 for slope dip up to $70^{\circ}$ and is partially stable which can be made stable with corrective support measures in the form of systematic bolting/ anchors or systematic shotcrete etc. In the field, it is found that with these measures rock slopes are stable for slope dip up to about $69^{\circ}$. Thus, it may be concluded that for Joint set 2 in the left abutment, predominant joint dip is $70^{\circ}$ to $75^{\circ}$. Rock in the right bank of river Pare is better compared to the right bank and falls under partially stable category with $40 \%$ failure probability.

\section{CONCLUSION}

The Pare Rock mass is found to be characterized by three joint sets both at the right and the left bank at the proposed dam site of Pare Hydro Electric Power project. In the left bank, during geological mapping, it was observed that Joint set 2 has joint dip ranging from $45^{\circ}$ to $75^{\circ}$. Joints having $45^{\circ}$ joint dip renders the rock slopes with $45^{\circ}$ slope dip and steeper completely unstable. However, at the site it has been found that rock slopes up to about $70^{\circ}$ stand albeit with certain support measures, but are not completely unstable. This may be because the predominant joint dips of joint set 2 are close to $75^{\circ}$.

Present study shows that the right rocky abutment is partially stable having $40 \%$ failure probability, thus can be regarded as normal rock slopes. Left rocky abutment is found to be completely unstable with $90 \%$ failure probability. 


\section{ACKNOWLEDGMENT}

This work is the result of a research project sponsored by NEEPCO Ltd. We sincerely acknowledge NEEPCO Ltd. for providing us the various geological as well as geotechnical data of Pare rock mass, which was necessary for carrying out the present work.

\section{REFERENCES}

[1] B. Singh, R. K. Goel, Book on "Engineering Rock Mass Classification", pp. 231-238.

[2] N. Barton, R. Lien, \& J. Lunde, "Engineering classification of rock masses for the design of rock support". In Rock mechanics (Vol. 6, pp. 189-236). New York: Springer-Verlag, 1974.

[3] Z.T. Bieniawski, "Engineering rock mass classification". New York: John Wiley \& Sons, 1989, p 251.
[4] D.H. Laubscher, "A geomechanics classification system for the rating of rock mass in mine design", Journal of the Southern African Institute of Mining and Metallurgy, Vol. 90, 1990 pp. 257-273.

[5] Z.T. Bieniawski, "Engineering classification of jointed rock masses," Civil Eng. S. Afr. 1973, 15, pp. 333-343.

[6] B. Singh et al., "A new slope mass rating in mountainous terrain, Jammu and Kashmir Himalayas: application of geophysical technique in slope stability studies" Landslides 10, 2013, pp. 255-265.

[7] M. Romana , "A geomechanical classification for slopes: Slope Mass Rating,” Universidad Politecnica Valencia, Spain, pp. 575-600.

[8] M. Romana, R. Tomás, J.B. Seron, "Slope Mass Rating (SMR) geomechanics classification: thirty years review", ISRM Congress 2015 Proceedings - International Symposium on Rock Mechanics, Quebec, Canada, May 10 to 13 2015. ISBN: 978-1926872-25-4, 10 pp.

[9] R. Hack, D. Price, and N. Rengers, "A new approach to rock slope stability - a probability classification (SSPC)," Bull. Eng. Geol. Env. 2003, 62, pp. 167-184. 